\title{
Mixture Effects in FIR Low-Pass Filtered Signals
}

\author{
J. Chiverton, Student Member, IEEE, and K. Wells
}

\begin{abstract}
Accurate classification of signals composed of two or more classification classes (e.g. biomedical imaging data with pathological structures) might utilize a density that takes account of the signal acquisition process. A new density based on a Gaussian PSF and another utilizing a phenomenological observation known as Benford's Law are presented. Histograms of filtered signals are compared with these densities. The results suggest that the Gaussian PSF based density is somewhat better than the Benford's Law density. Both approaches provide improved fits to histograms from data convolved with a variety of different PSFs over an existing mixture formulation.

\section{EDICS}

SAS-STAT, IMD-MDSP, BIO-BIOL

\section{CONTACT DETAILS}

Dr. Kevin Wells,

Centre for Vision, Speech and Signal Processing,

School of Electronics and Physical Sciences,

University of Surrey, Guildford,

Surrey, GU2 7XH, UK

j.chiverton@surrey.ac.uk Tel. (+44)1903717778 Fax. (+44)1483686031

k.wells@eim.surrey.ac.uk Tel. (+44)1483686036 Fax. (+44)1483686031

\section{Index Terms}

mixture prior density, partial volume, mixed pixel, Benford's Law, statistical signal analysis 


\section{Mixture Effects in FIR Low-Pass Filtered Signals}

\section{INTRODUCTION}

HIS work is concerned with the estimation of prior mixture distributions used in the statistical analysis of
signals that have been significantly affected by a low-pass Finite Impulse Response (FIR) process. Exemplar applications where such signal analyzes are undertaken include the statistical classification of 3-D biomedical based image data [1], 2-D satellite based image data [2] and alpha matte computation in video data [3]. The signal acquisition process often affects the resultant data so that the signal magnitude close to an edge or boundary may change due to blurring of the signal levels from the FIR filtering operation of the signal acquisition process, thus making classification and quantification more complicated. To illustrate this, if one is concerned with the size of a tumor located within a medical image, then prior knowledge of the governance of the true underlying signal affected by the acquisition process will allow more accurate calculation of the probabilities of tumor presence in individual voxels in the data space.

This work presents a new method for modeling the mixture prior density found in data samples, (e.g. pixels or voxels) given a system Point Spread Function (PSF). It also relates an old phenomenological observation, namely Benford's Law, to the effects of the signal acquisition process, common to many signal acquisition devices.

A low-pass FIR process, when applied to a signal, produces blurring in the data. This blurring might occur as a result of a signal acquisition process and is sometimes known as the Partial Volume (PV) effect in 3-D data or mixed pixels (mixels) in 2-D image data. For the classification of such data, statistical analysis might utilize the posterior density given Bayes' theorem:

$$
p(\boldsymbol{\alpha} \mid x)=\frac{p(x \mid \boldsymbol{\alpha}) \cdot p(\boldsymbol{\alpha})}{p(x)}
$$

where $p(\boldsymbol{\alpha})$ is the prior mixing density for which $\boldsymbol{\alpha}=\left(\alpha_{1}, \ldots, \alpha_{m}\right)^{T}$ represents the amount of mixing that a single signal element (such as a pixel) possesses for $m$ components. $p(x)$ represents the marginal density for the measured signal and $p(x \mid \boldsymbol{\alpha})$ is the likelihood of obtaining a particular signal value given a set of particular mixture values.

This work is concerned with the underlying prior mixing density, $p(\boldsymbol{\alpha})$. Linear mixing is assumed, so that, for two 
classes, $j$ and $k, \alpha_{j}=1-\alpha_{k}$. This means $\boldsymbol{\alpha}$ can be fully specified as a scalar value, $\alpha$, resulting in a single-variate prior probability, $p(\alpha)$. Initially $p(\alpha)$ is estimated analytically. The result of this analysis is then compared with the proposed application of Benford's Law. The applicability of these new results are then demonstrated on synthetic volumetric image data, where the true prior densities are known.

\section{THEORY}

\section{A. Gaussian Based Derivation of the Prior Density}

If one considers an idealized bimodal signal to be characterized by a step edge composed of an infinite number of frequencies, then the result of a band-limited signal acquisition process with a low-pass frequency FIR will smooth the step-edge, thus reducing the higher-frequency components in the signal. To illustrate this, an idealized noiseless edge with intensity values arbitrarily assigned to values of 0 and 1 can be described by:

$$
f(x)=\left\{\begin{array}{c}
1 \text { for } \quad x>0 \\
0 \text { for } x \leq 0
\end{array} .\right.
$$

If the low-pass FIR of the signal acquisition process can be characterized by a Gaussian PSF, $h(x)$, (as often found in image or signal acquisition systems), then equation 2 can be convolved with this kernel to obtain the idealized noiseless representation of the signal post-acquisition:

$$
\begin{aligned}
y(x) & =\int_{-\infty}^{\infty} f(\tau) \cdot h(x-\tau) \cdot d \tau \\
& =\int_{0}^{\infty} \frac{1}{\sqrt{2 \cdot \pi \cdot \sigma^{2}}} \cdot \exp \left(-\frac{(x-\tau)^{2}}{2 \cdot \sigma^{2}}\right) \cdot d \tau .
\end{aligned}
$$

The result of this convolution, $y(x)$, is given by evaluating equation 3 resulting in:

$$
y(x)=1 / 2\left(\operatorname{erf}\left(x / \sqrt{2 . \sigma^{2}}\right)+1\right)
$$

where $\operatorname{erf}(t)=\frac{2}{\sqrt{\pi}} \int_{0}^{t} \exp \left(-u^{2}\right) \cdot d u$ is the Gaussian error function. It is now desirable to obtain a continuous histogram model of the signal represented by equation 4 . This can be considered as a function that describes the proportion of the signal that occupies finite ranges of $y(x)$. It can be determined by initially finding the inverse of $y(x), x(y)=y^{-1}(x)$ and then dividing this inverse function into intervals, thus:

$$
x(y)=\sqrt{2 . \sigma^{2}} \cdot \operatorname{erf}^{-1}(2 . y-1)
$$


where $\operatorname{erf}^{-1}(t)$ is the inverse error function, so that $\operatorname{erf}\left(\operatorname{erf}^{-1}(t)\right)=t$.

The frequency information of $y(x)$ can be found by determining the distance $x(y)$ travels in a small interval, represented by $D(y) \approx x(y+\delta y)-x(y)$, and taking the limit of $\delta y \rightarrow 0$ results in the first derivative of $x(y)$, $D(y) \approx \frac{\mathrm{d} x(y)}{\mathrm{d} y}$. Utilizing the result of Carlitz [4], the first derivative of the inverse error function is taken to be $\frac{\operatorname{derf}^{-1}(z)}{\mathrm{d} z}=\frac{\sqrt{\pi}}{2} \exp \left(\operatorname{erf}^{-1}(z)^{2}\right)$, and letting $\alpha=y$ so that:

$$
\begin{aligned}
& p_{\text {Gauss }}(\alpha=y)=\left.C_{G_{j, k}} \cdot \frac{\mathrm{d} x(y)}{\mathrm{d} y}\right|_{y=\alpha} \\
& =C_{G_{j, k}} \cdot \sqrt{2 \cdot \pi \cdot \sigma^{2}} \cdot \exp \left(\operatorname{erf}^{-1}(2 . \alpha-1)^{2}\right),
\end{aligned}
$$

where $C_{G_{j, k}}$ is a normalizing term for the above Gaussian based model. This result illustrates that the standard deviation of the original convolution PSF, $\sigma$, has no affect on the shape of the resulting mixture density other than to contribute a scaling parameter. This Inverse Cumulative Gaussian (ICG) density is illustrated in figure 1.

\section{B. Benford's Law}

A phenomenological law, known as Benford's Law, has previously been used to describe the natural ordering of frequency data. Frank Benford originally discovered this phenomenological law in 1938. Benford initially suggested the following equation [5]:

$$
B(\beta)=\log _{10}\left(1+\frac{1}{\beta}\right)
$$

where $\beta=(0,10)$. Benford observed the applicability of this law in numbers taken from newspapers, atomic weights and black body radiation. This law can be extended to any number of digits, $\boldsymbol{\beta}=\left\{\beta_{1}, \ldots, \beta_{Q}\right\}$, with specific order, by the following [5], [6]:

$$
B(\boldsymbol{\beta})=\log _{10}\left[1+\left(\sum_{q=1}^{Q} \beta_{q} \cdot 10^{Q-q}\right)^{-1}\right] .
$$

Hill in 1996 [6], provided an explanation of Benford's Law by showing that if data comes from many different distributions and is scale and base invariant then the overall distribution will tend towards Benford-like behavior. Similarly, the result of the FIR filtering process on noiseless data produces data points that are governed by many different distributions due to the large number of different edge or boundary configurations and the result of their convolution with the PSF. The result of this filtering operation also produces histograms that are scale invariant 
(the histograms are not dependent on the size of the pixels or voxels) and base invariant (independent of the number of signal levels between signal components $j$ and $k$ ). As a result, one can consider the prior mixing density to be composed of two equal, but symmetrically opposite ordered frequency components that are not independently directly observable. If their range is $(0,1)$ then they are reflected about 0.5 . Such a single ordered frequency component relates the idealized (no-noise) low-pass filtered signal plotted against its relative frequencies, for component $j$ :

$$
p\left(\alpha_{j}\right)=C_{B_{j}} \cdot \log _{10}\left(1+\frac{1}{\mu_{j}}\right)
$$

where $j$ is indicative of a particular signal component under consideration and $C_{B_{j}}$ is a normalizing factor. The scaled mixing term, $\mu_{j}$, is given by $\mu_{j}=\operatorname{int}\left(\left(1-\alpha_{j}\right) \cdot 10^{p}+0.5\right)$ where $p$ is the precision, int () results in the integer part of the argument and $\alpha_{j}=(0,1)$. If the second signal component, $k$, is also considered, then the joint probability results in:

$$
\begin{aligned}
& p\left(\alpha_{j}, \alpha_{k}\right)=C_{B_{j, k}} . \\
& \left(\log _{10}\left(1+\frac{1}{\mu_{j}}\right)+\log _{10}\left(1+\frac{1}{\mu_{k}}\right)\right),
\end{aligned}
$$

where $C_{B_{j, k}}$ is a normalizing term. This result is similar to the previously defined ICG density in equation 6 , in that equation 10 is scale invariant, i.e. there is no parameter to control the shape of the density. This Benford prior density is also illustrated in figure 1.

\section{EXPERIMENTAL Methodology}

A $\chi^{2}$ Goodness-of-Fit (GoF) test of the two models described thus far, $p_{\text {Gauss }}(\alpha)$, the ICG prior density and $p_{\text {Benford }}(\alpha)$, the Benford prior density and a further density given in [2] has been performed on histograms produced from simulated 3-D data. The simulated data consists of concentric spheroids with alternating intensities, $j$ and $k$, filtered with low-pass FIR filters designed using the Kaiser window method [7] to model a variety of FIR processes.

\section{A. FIR Filter Design}

The Kaiser window method calculates the signal domain filter coefficients, $w_{\delta}$ by:

$$
w_{\delta}(n)=\frac{1}{I_{0}(\delta)} \cdot I_{0}\left(\delta \cdot \sqrt{1-\frac{2 . n}{N-1}}\right)
$$


where $n=\left[-\frac{N}{2}, \frac{N}{2}\right]$ is the co-efficient index. $N$ is the window size and $\delta$ is used to express the maximal ripple or side lobe magnitude as a fraction of the maximal pass band gain. $I_{0}(u)$ is the modified Bessel function of the first kind, order $0 . N$ and $\delta$ are calculated according to a well known algorithm (see for example [8]), where a further parameter, the transition width, $D$, is required. This algorithm therefore enables us to design a low-pass FIR filter with a variety of frequency and signal domain properties. The parameters of the windows that were designed for this work can be seen in table $\mathrm{I}$, where the transition width, $D$, is expressed as a fraction of the sampling rate.

For the first row in table I, i.e. $\delta=9.0 \mathrm{E}-02$, the filter function is equivalent to a boxcar [8]. A boxcar filter is often used in synthetic data, such as computer graphics or simulation of the PV effect [9].

The fifth row in table I, i.e. $\delta=1.1 \mathrm{E}-03$, is closest to a Hamming window, $w(n)=0.54+0.46 \cdot \cos (\pi \cdot n / N)$, [8]. For the filter functions in rows 6 to $9(\delta>1.1 E-03)$, the Kaiser window can be thought to approximate a Gaussian window, which is a commonly assumed PSF in many imaging and signal processing applications. Figure 2 illustrates the Kaiser window function in the signal intensity domain, for the above three examples representing the boxcar, Hamming and Gaussian window functions.

\section{B. Prior Density Evaluation}

The densities evaluated were the ICG mixture prior density given by equation 6; the Benford mixture prior density given by equation 10; and for comparison, the Kitamoto Density [2]:

$$
p_{K i t a}\left(\alpha_{j}\right)=-\frac{4 \cdot c_{k}^{2}}{\left(b_{k}+c_{k}\right)^{2}} \cdot \log \left(\alpha_{j}\right),
$$

where Kitamoto defined $c_{k}$ as the size of a pixel and $b_{k}$ as the size of the foreground object that has been subjected to a smoothing process. For this particular density, Kitamoto asserted that the pixel size should be smaller than the foreground object size, i.e. $b_{k}>c_{k}$. An observation with regards to this particular density is the fact that the shape of the density is not affected by the parameters of the imaging process, i.e. $c_{k}$ and $b_{k}$, similar to the densities proposed in this work (equations 6 and 10). However, as can be seen from figure 1, the densities possess different shapes in the tail regions and consequently different probabilities in the center region of each density, when fitted to FIR filtered data. 


\section{RESULTS}

An exemplar set of data points drawn from histogram data smoothed by a Kaiser window with a transition width, $D=0.305$ and maximum ripple size, $\delta=1.4 E-05$, can be seen in figure 1 . This figure also plots the three theoretical densities. The $\chi^{2}$ GoF results seen in figure 3, illustrate that the ICG prior density possesses the lowest $\chi^{2}$ errors with respect to the Kaiser filtered data for the majority of cases. One can also observe that that the ICG error decreases as the filter functions tend towards a Gaussian window function.

The Benford prior density also results in smaller $\chi^{2}$ error terms when compared with the Kitamoto prior density and appears to closely follow the ICG prior density $\chi^{2}$ term, although every Benford error is greater than the corresponding ICG error.

The GoF error for all three prior models significantly increases under two circumstances. The first circumstance being when the window filter function is significantly larger than the objects of interest in the data. The concentric spheroid bands in the simulated data have a finite width, and for particular sets of parameters for the Kaiser window method, the window size is significantly larger than the widths of the bands. These occur from the lower left of the windows in table I, thus for these cases, the error is significantly greater.

The second circumstance for large GoF errors for all three models is when the window filter function becomes very small (less than 3 pixels wide). The result of a very small window is to cause digitization effects on the resulting densities where a large number of the bins in the digital histograms are empty, thus contributing to a larger error.

\section{Discussion AND CONCLUSIONS}

This investigation into theoretical prior densities for mixed signal problems is limited to cases where: the width of the kernel for the acquisition process is smaller than the objects of interest affected by the mixing process; and the PSF can be approximated by one of the FIR window filters designed using the Kaiser window method.

A methodology for modeling a density from the signal intensity domain PSF has been presented. This has been illustrated with a Gaussian PSF model resulting in improved $\chi^{2}$ GoF for PSFs that approach a Gaussian. This investigation has also high-lighted that the Benford prior density can be used as a plausible description of the 
mixing that results from signal processes characterized by a FIR filtering process using a finite window width. Each of the theoretical prior mixing densities possess functional shape invariance given different sized window functions. These new densities could find application in statistical analyzes of signals obtained with low-pass band-limited signal acquisition processes.

\section{ACKNOWLEDGMENT}

The authors would like to acknowledge support from the UK EPSRC for funding this work. They would also like to thank Dr. Theodore Vlachos at the University of Surrey and the reviewers for their helpful comments.

\section{REFERENCES}

[1] J. Chiverton, K. Wells, M. Barry, M. Partridge, H. Kadhem, and R. Ott, "The partial volume effect in pet/spect and benford's law," in Proc. IEEE NSS and MIC, Puerto Rico, Oct. 2005.

[2] A. Kitamoto and M. Takagi, "The area proportion distribution-relationship with the internal structure of mixels and its application to image classification,” Sys. Comps. Japan, vol. 31, pp. 57-76, 2000.

[3] Y. Wexler, A. Fitzgibbon, and A. Zisserman, "Bayesian estimation of layers from multiple images," in Proc. ECCV, Copenhagen, Denmark, 2002, pp. 487-501.

[4] L. Carlitz, "The inverse of the error function,” Pacific J. Math, vol. 13, pp. 459-470, 1963.

[5] F. Benford, "The law of anomalous numbers," Proc. Amer. Phil. Soc., vol. 78, pp. 551-572, 1938.

[6] T. Hill, “A statistical derivation of the significant-digit law," Stat. Sci., vol. 10, pp. 354-363, 1996.

[7] J. F. Kaiser, "Digital filters," in System Analysis by Digital Computer, F. Kuo and J. Kaiser, Eds. New York, USA: Wiley, 1966.

[8] E. Ifeacher and B. Jervis, Digital Signal Processing A Practical Approach. Addison-Wesley, 1993.

[9] J. Links, L. Beach, B. Subramaniam, M. Rubin, J. Hennessey, and A. Reiss, "Edge complexity and partial volume effects," J. Comput. Assist. Tomogr., vol. 22, pp. 450-458, 1998. 


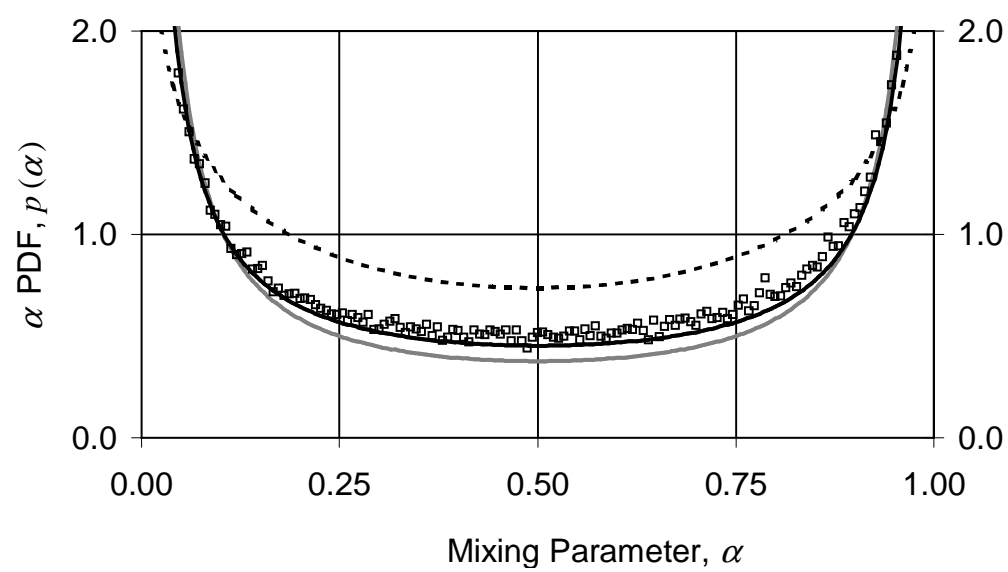

Fig. 1. Comparison of the three theoretical prior mixing densities, Benford mixing density (gray line), Kitamoto mixing density (dotted line) and the ICG mixing density (black line). Exemplar data points are also shown (squares) for the simulated data smoothed with a transition width, $D=0.305$ and maximum ripple size, $\delta=1.4 E-05$. These exemplar data points are typical of the data points produced via the smoothing effect of the FIR filtering process.

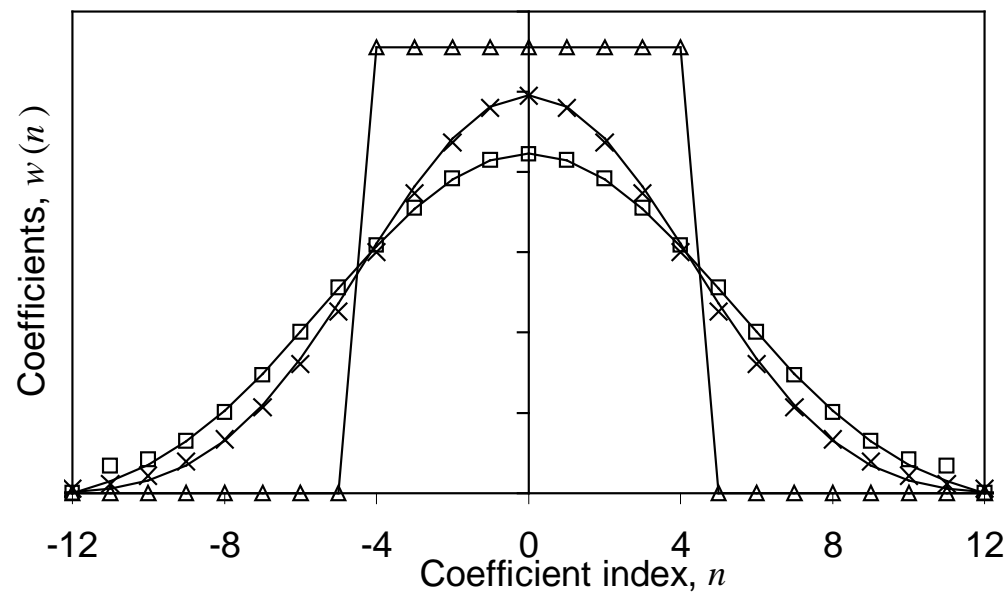

Fig. 2. Three window functions (lines) and their approximations using the Kaiser window design method (points superimposed). The Boxcar window is given by the line joining the triangles; the Hamming window is given by the smooth line joining the squares; and a Gaussian window joining the crosses. 

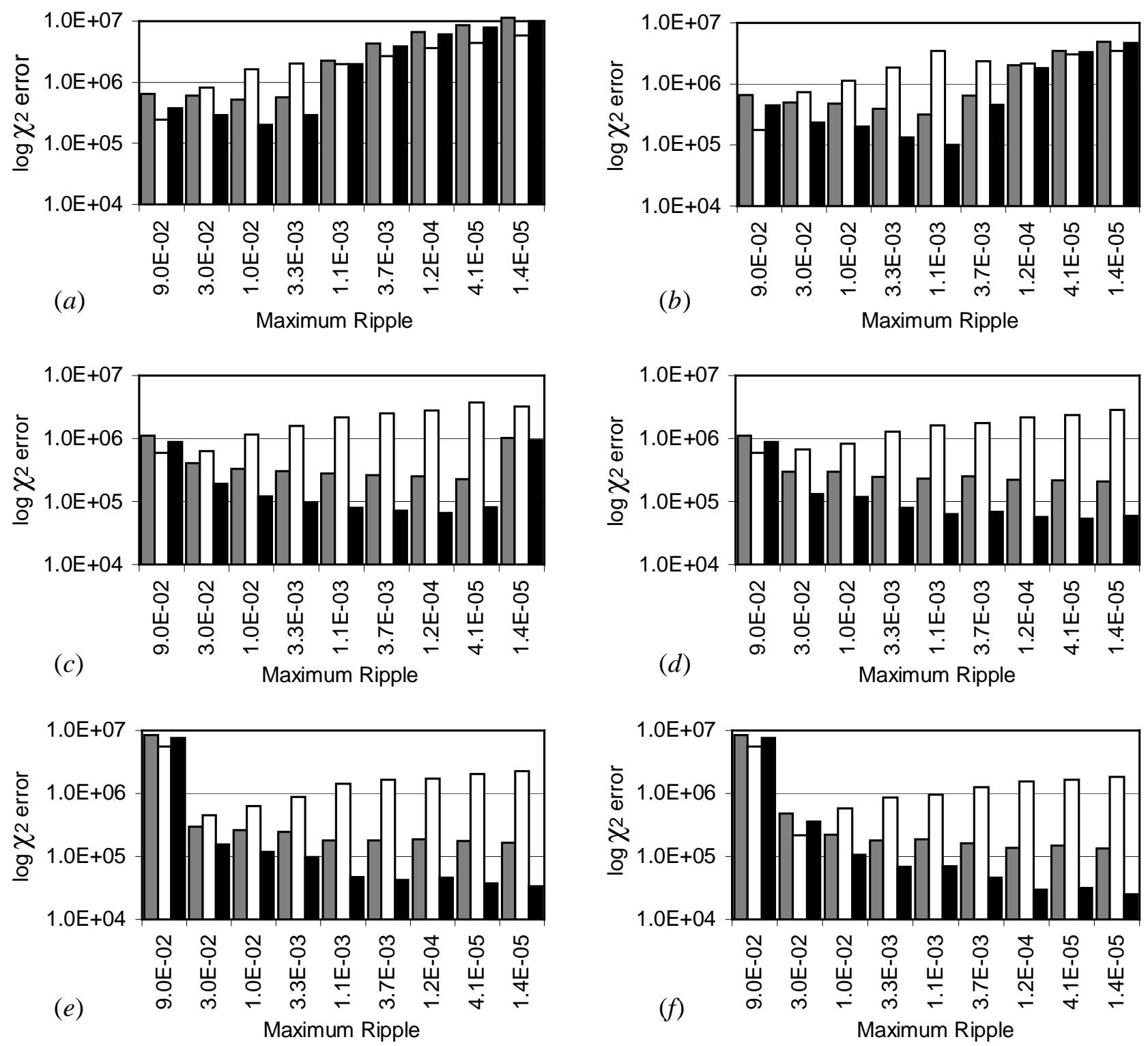

Fig. 3. $\chi^{2}$ GoF results for Benford (gray), Kitamoto (white) and Gaussian (black) prior mixture densities when compared with the experimental mixture densities obtained by convolving simulated volumetric data sets with window functions designed using the Kaiser window design methodology with parameters given in table I. (a) is for transition width, $\mathrm{D}=0.100$; (b) is for transition width, $\mathrm{D}=0.125$; (c) is for transition width, $\mathrm{D}=0.156$; (d) is for transition width, $\mathrm{D}=0.195$; (e) is for transition width, $\mathrm{D}=0.244$; and (f) is for transition width, $\mathrm{D}=0.305$. 
TABLE I

Kaiser Window Parameter Values: Window Widths, $N$ FOR A GIVEn RipPle VAlue, $\delta$ (Fraction OF MAXIMUM PASS BAND GAIN) AND TRANSITION WIDTH, $D$,(FRACTION OF SAMPLE RATE).

\begin{tabular}{|c||c||c||c||c||c||c|}
\hline \multicolumn{1}{|c||}{ Ripple } & \multicolumn{6}{c|}{ Transition Widths, $D$} \\
\hline$\delta$ & 0.100 & 0.125 & 0.156 & 0.195 & 0.244 & 0.305 \\
\hline $9.0 \mathrm{E}-02$ & 9 & 7 & 6 & 5 & 4 & 3 \\
$3.0 \mathrm{E}-02$ & 16 & 13 & 10 & 8 & 6 & 5 \\
$1.0 \mathrm{E}-02$ & 22 & 18 & 14 & 11 & 9 & 7 \\
$3.3 \mathrm{E}-03$ & 29 & 23 & 19 & 15 & 12 & 9 \\
$1.1 \mathrm{E}-03$ & 36 & 28 & 23 & 18 & 15 & 12 \\
$3.7 \mathrm{E}-03$ & 42 & 34 & 27 & 22 & 17 & 14 \\
$1.2 \mathrm{E}-04$ & 49 & 39 & 31 & 25 & 20 & 16 \\
$4.1 \mathrm{E}-05$ & 56 & 44 & 36 & 28 & 23 & 18 \\
$1.4 \mathrm{E}-05$ & 62 & 50 & 40 & 32 & 25 & 20 \\
\hline
\end{tabular}

